\title{
The Development of Prediction Indicators on Currency Market Using Neuro-Fuzzy Method
}

\author{
Ronald Limowa Lie ${ }^{1,}$ Murtiyanto Santoso ${ }^{1}$, Felix Pasila $^{1}$, Raymond Sutjiadi ${ }^{2}$, \\ and Resmana Lim, ${ }^{1, *}$ \\ ${ }^{1}$ Electrical Engineering Department, Faculty of Industrial Technology, Petra Christian University, \\ Jl. Siwalankerto No.121-131, Surabaya, East Java, 60236 Indonesia \\ ${ }^{2}$ Informatics Department, Faculty of Information Technology, Institut Informatika Indonesia, \\ Jl. Pattimura No.3, Surabaya, East Java, 60189 Indonesia
}

\begin{abstract}
A technical indicator is an analysis instruments to help traders analyzing forex price movements through charts. Prediction indicators are artificial technical indicators that can help traders to analyse forex price movements in the future. This prediction information becomes one of the bases in making trading decisions. This project aims to develop prediction indicators on MetaTrader that can provide information on forex price predictions using Neuro-Fuzzy method. The Neuro-Fuzzy System requires input parameters in the system prediction process obtained from the system training process. These parameters can be through or without optimization process. The prediction indicator will also make trading decisions based on prediction indicators analysis, RSI, and Stochastic. Finally, the information on trading decisions will be displayed on Facebook pages. The prediction indicator testing run well on a trading system. Prediction indicators with parameters before optimization were well used in the H4 EURUSD pair (data 2012) with a predicted profit of USD 16 499. While the prediction indicators with parameters after optimization were well used in the $\mathrm{H} 1$ EURUSD pair with a predicted profit of USD 21 945. The information on trading decisions were also successfully displayed on Facebook pages.
\end{abstract}

Keywords: Artificial technical indicator, facebook, forex, forex price analysis, metatrader.

\section{Introduction}

Forex trading is a promising investment choice as it can be conduct-ed $24 \mathrm{~h}$ a day and $5 \mathrm{~d}$ a week. The forex market is so very crowded with many players that it is considered very liquid. Forex price movements are determined by market power and a country's economic conditions so it is very difficult to predict the direction of the forex price movements. When forex price movements is analyzed, a pattern or repetitive behavior will be found, called a trend. This trend can be studied and analyzed to form an indicator that can help traders making trading decisions [1].

\footnotetext{
*Corresponding author: resmana@petra.ac.id
} 
In daily trading, a trader must monitor the price movements through a chart, not to miss the trading momentum. Psychological factors also determine trading decision making. Therefore, it is necessary to make a prediction indicator using Neuro-Fuzzy method that can tell the trend of forex price movements in the future. This prediction indicator will be an add-on to MetaTrader [2-4].

\section{Project design and implementation}

The project consists of system training scripts and prediction indicators on Meta-Trader; Neuro-Fuzzy methods in the form of DLL files; and a Facebook application of status updates.

\subsection{System design}

The Neuro-Fuzzy method [5-7] being used, previously exists on Matlab. This method needs an interface to be able to be used on Meta-Trader. Takagi-Sugeno's fuzzy inference system is used for the Neuro-Fuzzy fuzzy method architecture as shown on Figure 1 below.

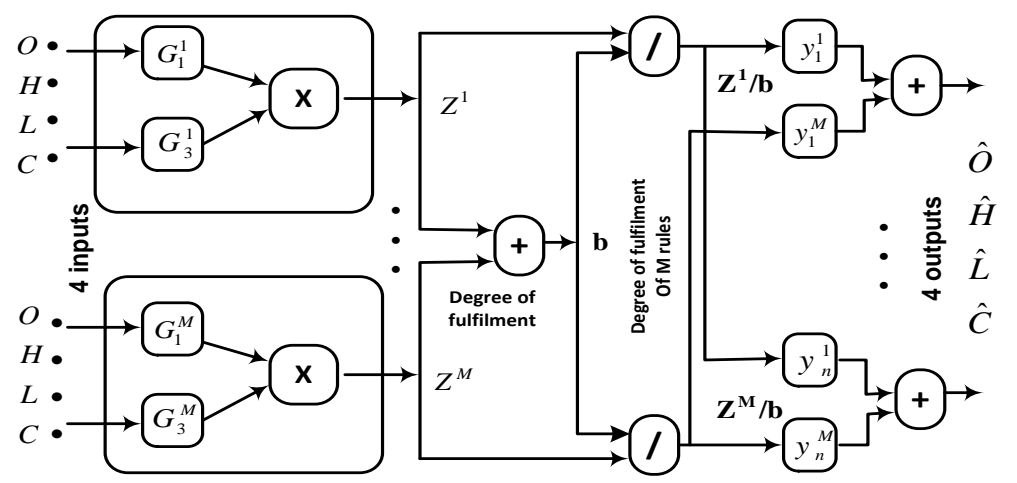

Fig. 1. Takagi-Sugeno fuzzy model with four inputs and four OHLC outputs (modification from Pasila structure, "Forecasting") [5].

Figure 1 shows that there are four inputs and outputs of the Neuro-Fuzzy system, consisting of the open, high, low, and close prices data of a pair forex. In the multiplier operator section $(\times)$, the input data will be compared to the $G$ parameter value representing the antecedent conjunction operator. The output of this section is the value of the degree of fulfilment corresponding to $1=1,2, \ldots, \mathrm{M}$ represents the number of rules. The divider section $(/)$, together with the addition operator $(+)$, calculates the normalized degree of fulfilment $\left(z^{l} / b\right)$ of each corresponding rule. After the multiplication with the corresponding Takagi-Sugeno consequent rule $\left(y_{j}^{l}\right)$, then used as an input for the final part of the addition $(+)$ on the defuzzified output value OHLC. This OHLC value becomes the input of the Neuro-Fuzzy system for the next iteration by the same working system $[6,8]$.

The Neuro-Fuzzy method is separated in the training and prediction phases. The goal is to separate the generated DLL files. The DLL system training file will be inputted into the system training script. The DLL prediction system file becomes the input to the prediction indicator. Figure 2 shows the Neuro-Fuzzy method algorithm, being used in the system training and prediction system. 


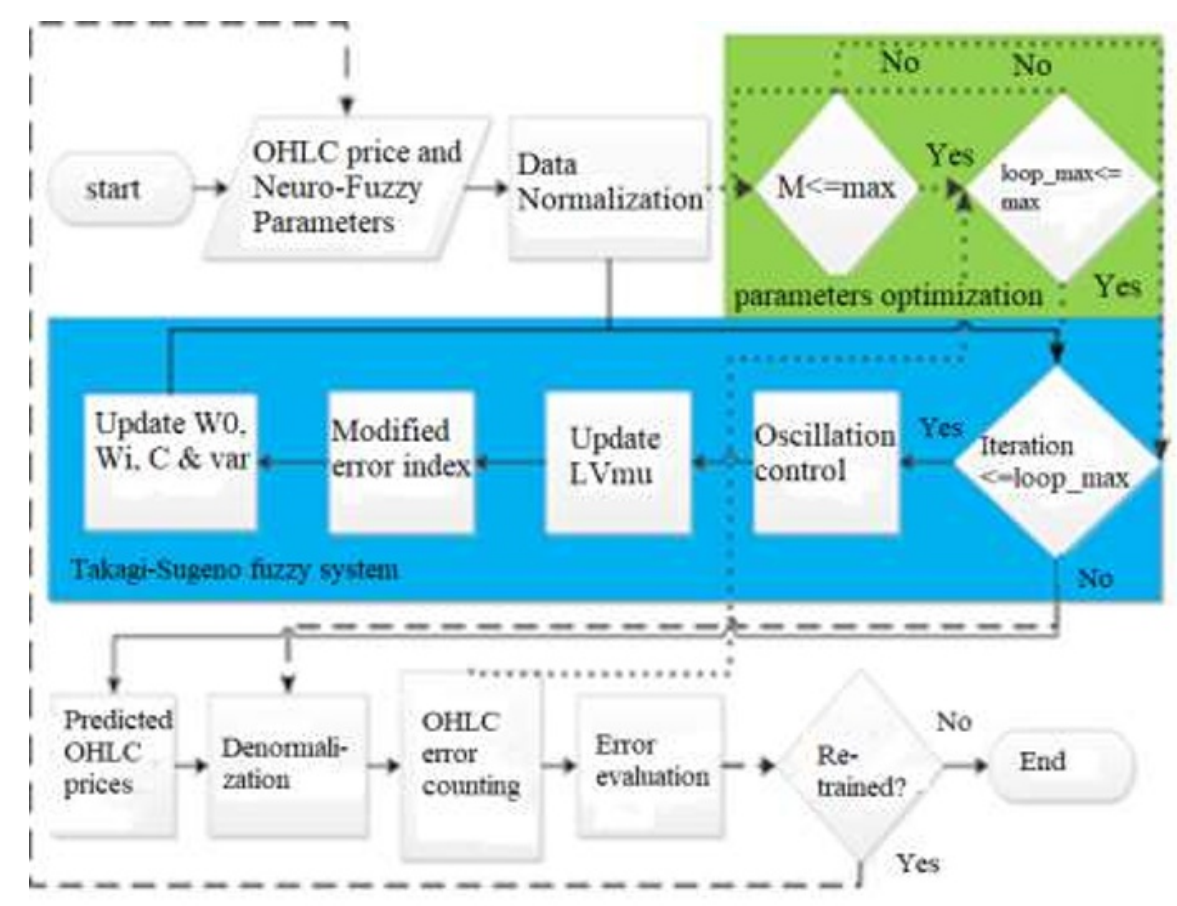

Fig. 2. Flowchart of the neuro-fuzzy working system.

System training scripts and prediction indicators are made on Meta-Trader. Both programs will run on the client terminal corresponding to their respective functions. To be able to run the prediction indicator program, it is necessary to do system training first. The aim is to get the parameters weight file as the result of the system training. Then the prediction indicator can run the prediction system by reading the input of the parameter weight file. In short, a system training system script called ANFIS_train can be described in accordance with the algorithm as follows:

i. Initializing the DLL file containing the Neuro-Fuzzy method as the input library and system training variables.

ii. Taking the history data of a pair forex and writing it in a CSV file as the system training input.

iii. Calling the system training function from the DLL file, called as the system training of the Neuro-Fuzzy method.

iv. Saving the result of system training in a CSV file.

Prediction indicators are also developed on the Meta-Trader client terminal. Prediction indicators can provide information on five time step forex price predictions in the future. Additional analysis can be obtained from technical indicators with the aim of further clarification on the accuracy of the predictions of forex price movements trend. The rules of each indicator are made in a trading system that will guide the trading decision making [9].

The RSI and Stochastic indicators are used as additional technical indicators in developing trading system rules. The trading system rules of the three indicators are as follows.

i. The order buy signals is given if the value of RSI $>45$, RSI $<50$, the value of the stochasticMain indicator $>$ stochasticSignal, and the difference of the prediction price on the chart used $>=$ threshold. 
ii. The order sell signal is given if the value of RSI $>50$, RSI $<55$, the value of the stochasticMin indicator $<$ stochasticSignal, and the difference of the prediction price on the chart used $<=$ threshold.

The trading system above is then applied in making prediction indicators called ANFIS_plot. The prediction indicator on Meta-Trader is based on the algorithm as follows:

i. Initializing the DLL file containing Neuro-Fuzzy method as the input library and prediction indicator variables.

ii. Checking the use RSIStochastic boolean variables as a reference to the trading system rules:

a. If yes, trading system is analyzed using prediction indicator rules, RSI, and Stochastic, and a trading signal is assigned in accordance with the fulfilled rules.

b. If not, waiting for the new bar.

iii. When a new bar appears, the prediction error data is written into a CSV file, then the time marker index condition is checked:

a. If fulfilled, the prediction error data is summed and written in a CSV file.

b. If it is not fulfilled, the history data of a pair forex is taken and written in a CSV file as the input of the prediction system.

iv. Calling the prediction system function from the DLL file, as the prediction system from the Neuro-Fuzzy method.

v. Describing the results of the prediction system on the chart in the form of indicator lines and dot objects as the system interface for the user.

vi. Re-checking the boolean use RSIStochastic variables as a reference to the trading system rules:

a. If not, the trading system is analyzed using the prediction rules indicator only, and a trading signal is assigned in accordance with the fulfilled rules.

b. If yes, the label object containing the last five errors data is described.

vii. Calculating and writing down the prediction error data of each bar in a CSV file as an evaluation for the Neuro-Fuzzy method.

The Facebook application consists of website and PHP application that will do the status update on the Facebook pages. The purpose of the website development is to authorize the users who want to use the Facebook application. The Facebook application authentication uses the OAuth (Open Protocol Authentication) protocol, aiming to gain user access rights in the form of token access [10]. This token access will be stored in a database for further usage when the application wants to access the user resources on Facebook.

The Facebook application in the form of PHP files will transfer the trading information messages from MetaTrader4 via e-mails to a Facebook page as a status update. PHP has the function of reading e-mails from a mail server with IMAP protocol. The status update function will use the Facebook SDK for PHP library [11]. Figure 3 shows the PHP application algorithm called PagesUpStatus as follows:

i. Initializing the Facebook configuration files and databases containing primitive functions.

ii. Connecting to the database to retrieve data

iii. Accessing the Token and numMail.

iv. Connecting to the mail server and then counting the number of mails in the INBOX directory.

v. Comparing the value of numMail and the number of mails in the INBOX directory:

a. If numMail $>=$ the number of mails in the INBOX directory, then the message "No new messages" is displayed and the connection to the database and mail server is closed. 
b. If numMail < the number of mails in the INBOX directory, then the contents of the latest mail message is retrieved and the API (AccessToken and mail messages) is executed to update the status on the Facebook page.

vi. Doing a query to change the numMail column data to be the same as the number of mails in the INBOX directory, then the connection to the database and mail server is closed.

vii. Delaying for $60 \mathrm{~s}$ and the process is restarted.

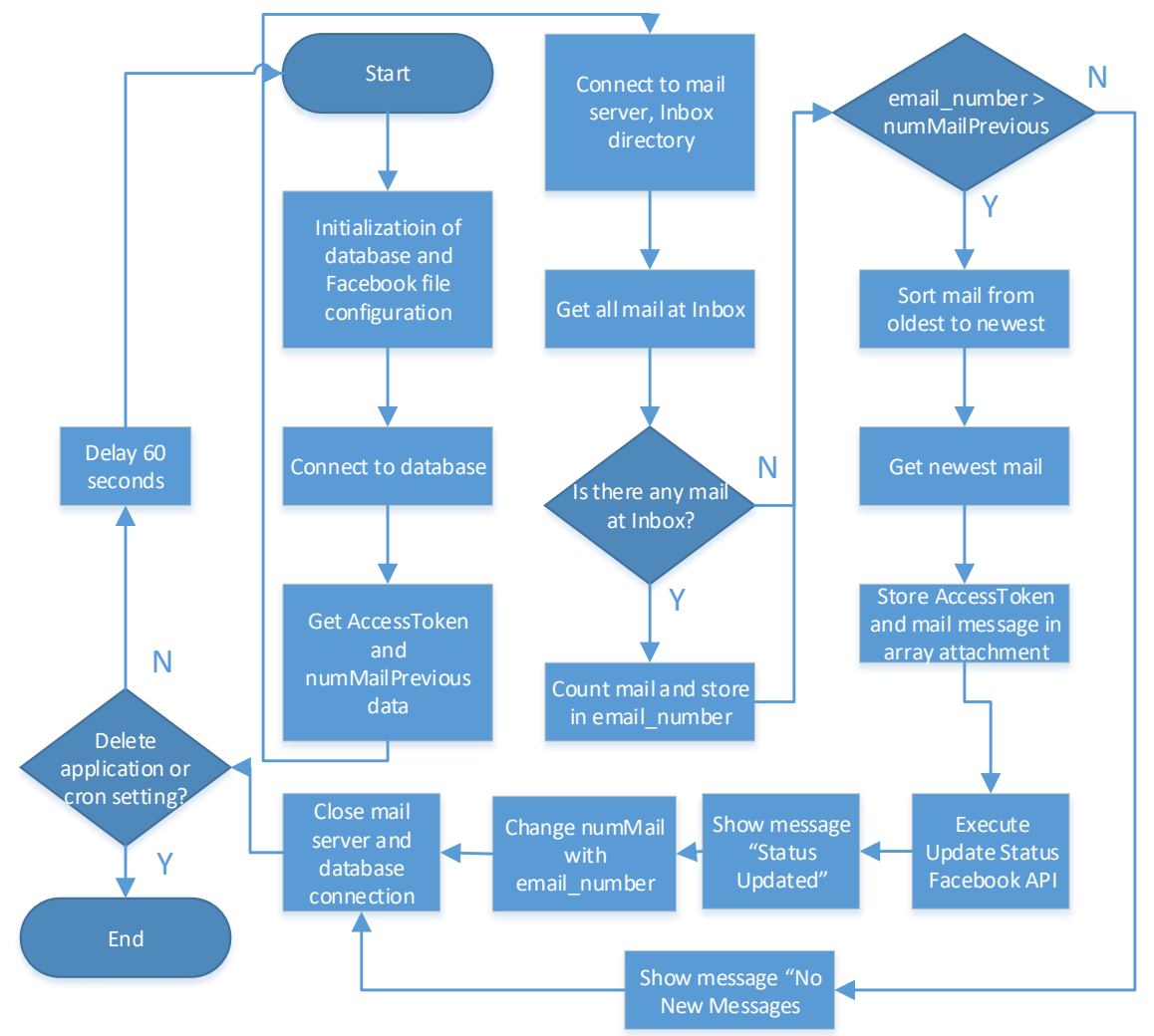

Fig. 3. Flowchart on the PagesUpStatus working application.

\subsection{System implementation}

The algorithms above become the basis for implementing the system in the form of programs. Each program will be developed in accordance with their respective placements. the implementation of the system is described as follows:

i. The Neuro-Fuzzy method is written from Matlab to the $\mathrm{C}++$ programming language, then compiled into DLL files. Two types of DLL files generated from the NeuroFuzzy method are DLL system training and DLL prediction system files. The DLL files will run the Matlab engine as a calculation engine for the Neuro-Fuzzy method. The DLL files will be the input library on the system training scripts and prediction indicators.

ii. The system training process is conducted at the Meta-Trader client terminal using the ANFIS_train and Matlab engine system training scripts as a calculation engine for the Neuro-Fuzzy method. The Neuro-Fuzzy Method conducts the system training based on the input data of a pair forex price and produces weight parameter files as the result 
of the system training. The weight parameter files will be saved corresponding to the name of the pair forex and timeframe used

iii. The prediction system process is also conducted on the Meta-Trader terminal client using the ANFIS_plot prediction indicators and the Matlab engine as a calculation engine for the Neuro-Fuzzy method. The Neuro-Fuzzy Method predicts the forex prices based on the input price of a pair forex and the weight parameter value of the previous system training. The result of the system prediction will be described on the Meta-Trader client terminal in the form of indicator lines and dot objects.

iv. The Facebook application will execute the API to update the status on the Facebook pages. The Facebook API requires an access parameter token, obtained from the users authentication of the Facebook application. The access tokens data and status update messages are executed by the Facebook application using the appropriate API. The status update messages on Face-book pages is the trading decisions information taken by the prediction indicators.

\section{Testing and system analysis}

The system testing consists of testing the accuracy of the trading system rules in providing the appropriate trading signals; testing the prediction indicators which consists of testing prediction indicator analysis transactions and calculating the prediction price errors; as well as testing the Facebook application to do the update status on Facebook pages. The NeuroFuzzy network are trained with 10 yr of data (2001 to 2011). Prediction indicator testing was con-ducted by backtesting using strategy tester feature on MetaTrader. The testing used EURUSD curency pair data from January to December 2012 on the M30, H1, H4, and D1 timeframes. Testings were also conducted using two types of parameters, before and after optimization.

\subsection{Testing of trading rules system}

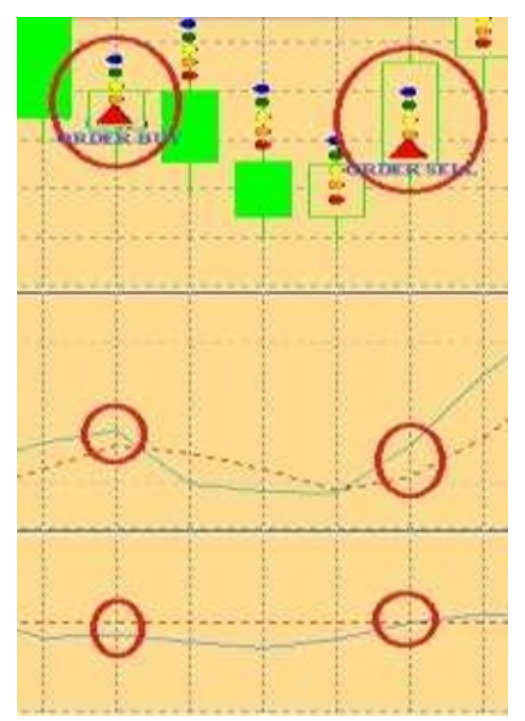

Fig. 4. Trading signal at client terminal.

This testing aims to analyze the appearance of trading signals on the MetaTrader client terminal, complying with the trading system rules analysis, whether fulfilled or not. The 
trading signals appearing on the client terminal were arrow objects, text objects, and the corresponding trading signal alerts, as well as an e-mail containing trading information to a user's e-mail address. At the appearance of a new bar, the previous trading signal was closed and the system performed a trading system rules analysis for the current bar. The testing result shows that the trading signals in the form of arrow objects, text objects, and alerts could be displayed on the client terminal in accordance with the fulfilled trading system rules analysis and a trading information e-mail was also sent to the user's e-mail address. Figure 4 shows an example of the results of the trading system rules testing.

\subsection{Prediction indicators transaction analysis testing}

The testing was conducted by measuring the accuracy of the trading decisions in trading simulations. It was conducted by calculating the amount of the profit/loss suffered due to the trading decision making. The amount of profit/loss suffered were compared between parameters before and after optimization on each month and testing timeframe. The purpose of this testing is to find out which parameters are of optimal use on certain timeframes with maximum benefit and the smallest risk.

The testing result shows that both prediction indicators with parameters before and after optimization could produce a good prediction system. This was proven by the results of the transactions which always record net profits at the end of the year for the M30, H1, H4 and D1 timeframes. The prediction Indicator with parameters after optimization was good to be used on $\mathrm{H} 1$ timeframe because it recorded the largest total net profit in a year compared to other timeframes with a small transaction risk as shown on Figure 5. While the prediction indicator with parameters before optimization was good to be used on the H4 timeframe because it recorded the largest total net profits of a year compared to other timeframes with a small transaction risk as shown on Figure 6.

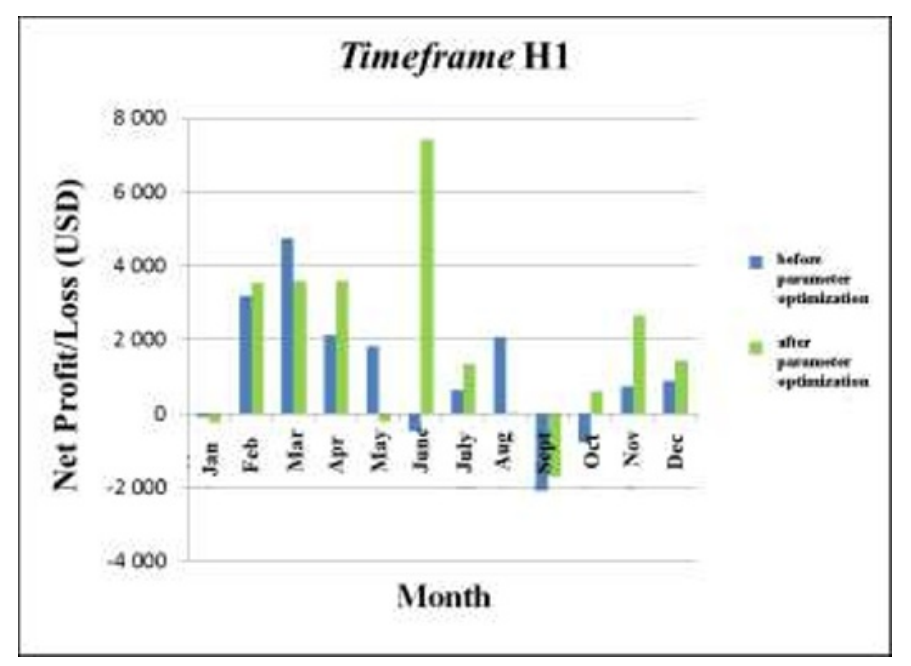

Fig. 5. Bar chart on the comparison of the testing result of transaction on timeframe H1. 


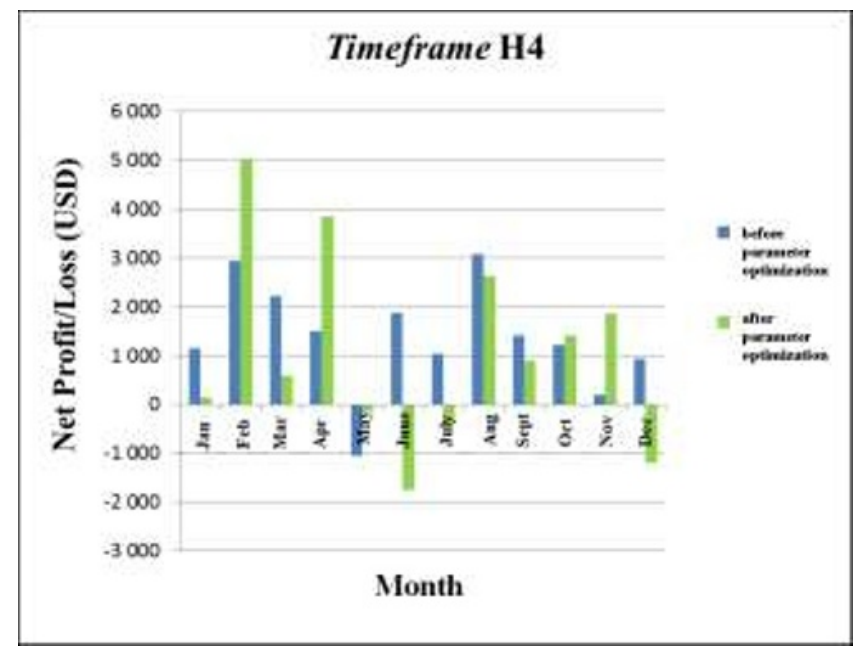

Fig. 6. Bar chart on the comparison of the testing result of transaction on timeframe H4.

\subsection{Calculation of price prediction errors}

Each prediction price must have errors or deviation towards the actual price. The amount of the prediction price errors was calculated and analyzed as a Neuro-Fuzzy system evaluation material. The calculation was performed on the five time step prediction price data in each bar in the form of the RMSE value (Root Mean Square Error). The calculation was performed on the close price data only at two types of parameters, before and after optimization. The amount of the prediction price errors between the two parameters were compared to find out which parameter could give the smallest price prediction error on a particular timeframe, appropriate to be used for the Neuro-Fuzzy system.

The calculation result shows that the prediction indicator with the parameter after optimization gave the smallest price prediction error on $\mathrm{H} 1$ timeframe. This result was also in line with the result of prediction indicator analysis transaction testing as shown on Figure 7. While the prediction indicator with the parameter before optimization gave the smallest price prediction error on the timeframe H4 as shown on Figure 8. This result was in line with the result of the pre-diction indicator analysis transaction testing with the parameter before optimization gave the largest total net profit on the $\mathrm{H} 4$ timeframe.

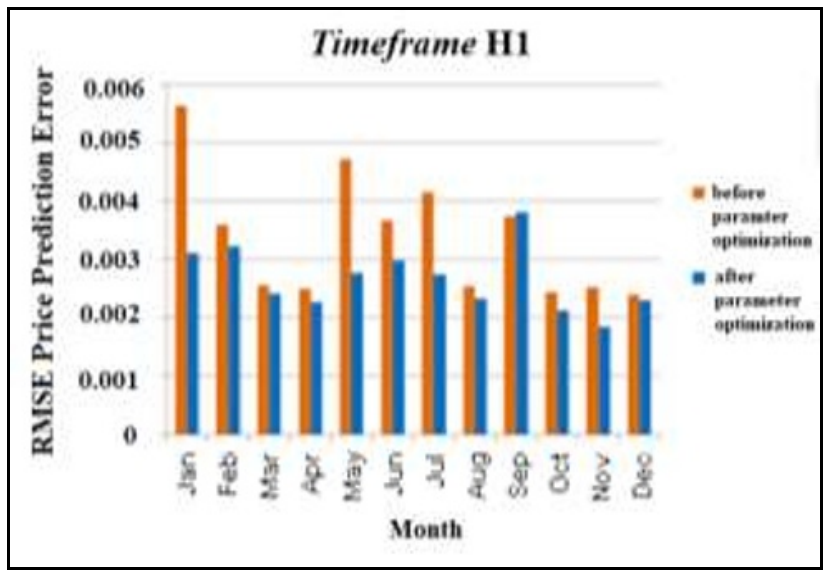

Fig. 7. The calculation result of price prediction errors on timeframe H1. 


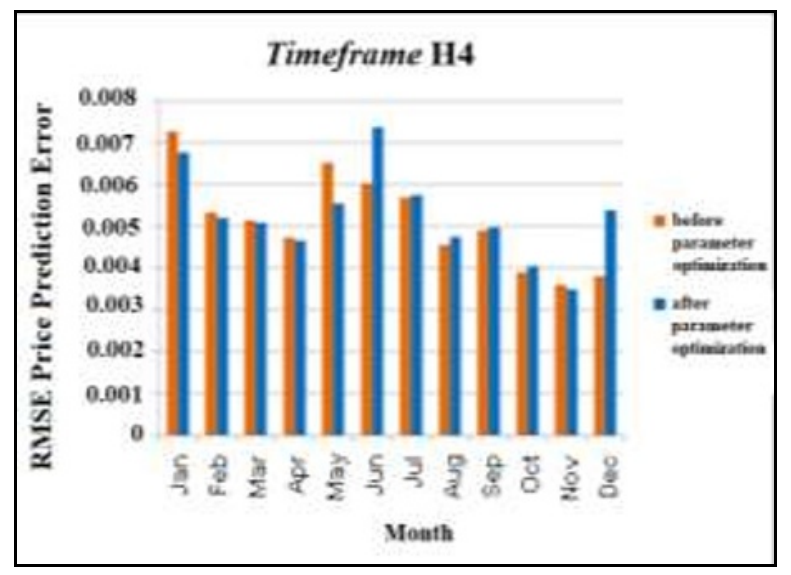

Fig.8. The calculation result of price prediction errors timeframe $\mathrm{H} 4$.

\subsection{Website Testing}

The Facebook application website consists of three pages: the home page, login page, and logout page. The users were directed to the Facebook login page after clicking the sentence containing a reference to the Facebook login page on the home page. After logging in and agreeing to the use of the application along with the permissions submitted, using authentication method, a user access right was obtained in the form of a token access. An access token is a user identification code which authorizes the use of the Facebook application.

The purpose of the website testing is to get access to the Facebook application user token and the changing AccessToken column and numMail data in the database. NumMail is a variable storing the number of mails in the user's INBOX directory. The testing result on Figure 9 shows the changes in AccessToken and numMail column data in the database corresponding to the current condition after the user logs out on the website page.

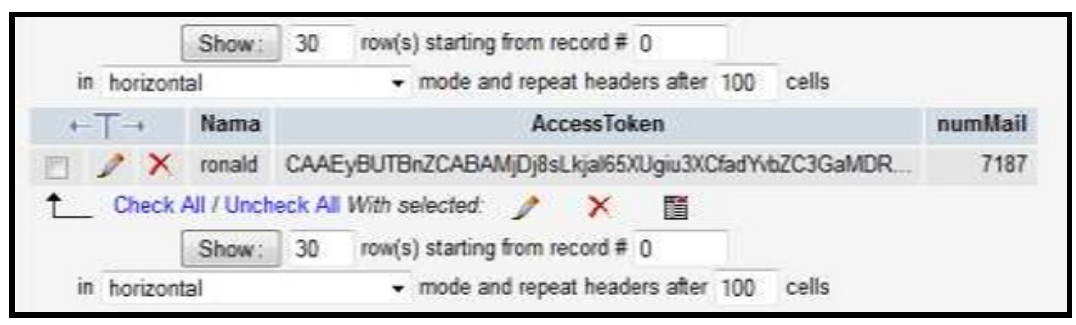

Fig. 9. Update column data in the database.

\subsection{PagesUpStatus application testing}

The PagesUpStatus application functions to transfer trading information messages from MetaTrader4 to a Facebook page via e-mails as an update status of the pages. The program was executed every $60 \mathrm{~s}$ in accordance with the settings of the cron command on the server.

The first step of the program was to retrieve AccessToken and numMail data from the database, then calculate the number of mails in the user's INBOX directory. The value was stored in a variable. Then the program compared the value of the numMail variable and the value of the number of mail variables in the INBOX directory. If the value of numMail was smaller than the number of mails in the INBOX directory, there would be a new mail in the 
INBOX directory. The program then retrieved the latest mail with the contents of the mail and executed the Facebook API to do the status on Face-book pages. If the value of numMail was greater than or equal to the number of mails in the INBOX directory, then there would be no new mail and the program would wait for $60 \mathrm{~s}$ to start the process again.

The testing result shows that the contents of the trading information message from MetaTrader to the e-mail address of the user could be displayed on the Facebook as an update status from the Facebok pages as shown on Figure 10. This status could also be displayed on the home page of each Facebook user who had previously liked those pages.

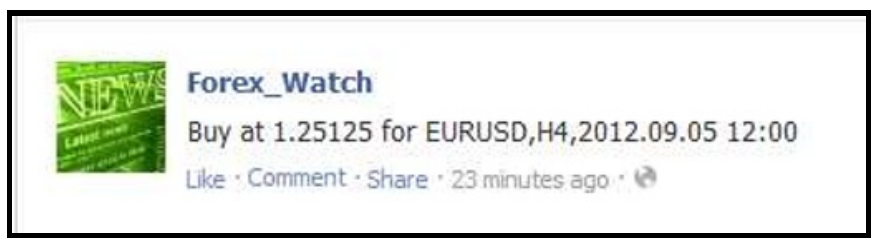

Fig. 10. An example of update status of Facebook pages.

\section{Conclusion}

Prediction indicators with both parameters before or after optimization can produce a good prediction system with a small transaction risk as it always generates net profit in a year in the M30, H1, H4, and D1 EURUSD timeframe pairs. Prediction indicators with parameters before optimization is good to be used on H4 EURUSD timeframe pair as it gives the largest total profit in a year, amounted to USD 16499 (data 2012). Meanwhile, prediction indicator with parameters after optimization is good to be used on the H1 EURSD timeframe pair as it provides the largest total profit in a year amounted to USD 21945 . The update status application on the Facebook pages runs well via the display of update status containing the trading decision information of the prediction indicator.

The authors are thankful to the grant (No. contract: 002/SP2H/LT/MONO/2019) provided by the Directorate of Research and Community Service, Ministry of Research, Technology, and Higher Education, Republic of Indonesia under grant scheme of Applied Research.

\section{References}

1. Babypips. What is a lot of forex? [online] from http://www.babypips.com/school/lotsleverage-and-profit-and-loss.html (n.d). [Accessed on August 20, 2020\}.

2. Babypips. Technical Analysis. [online] from http://www.babypips.com/school/ technical-analysis.html (n.d.). [Accessed on August 20, 2020].

3. A.K. Palit, D. Popovic. Computational intelligence in time series forecasting, theory and engineering applications. Verlag, London : Springer (2005). pp. 230-246. https://www.springer.com/gp/book/9781852339487

4. R. Babuska, H. Verbruggen. Annu. Rev. Control, 27,1:73-85(2003). https://www.sciencedirect.com/science/article/abs/pii/S1367578803000099

5. F. Pasila. Forecasting of electrical load using takagi-sugeno type mimo neuro-fuzzy network. [Thesis] University of Bremen, Germany (2006). https://scholar.google.com/scholar?cluster $=3693450068232213715 \& \mathrm{hl}=\mathrm{en} \&$ as $\_$sdt $=2$ $005 \&$ sciodt $=0,5$

6. F. Pasila, H. Kuswanto, H. Ferdinando, R. Sautama. Long-term prediction of IHSG Indonesia using levenberg-marquardt training on neuro-fuzzy network. Proceeding of 
New Trends and Development in Information Communication and Technology (ICT) in Creative Economy. (Jakarta, Indonesia, 2007). pp. 9-13(2007).

https://www.researchgate.net/publication/286379642_Long-term_Prediction_of

IHSG Indonesia using Levenberg Marquardt

7. F. Pasila, A.K. Palit, G. Thiele, G. JACIII, 12,4:361-369(2008).

https://www.fujipress.jp/jaciii/jc/jacii001200040361/

8. M. Santoso, R. Sutjiadi, R. Lim. MATEC Web Conf., 164,01031:1-5(2018).

https://www.matec-conferences.org/articles/matecconf/abs/2018/23/matecconf icesti2018 01031/matecconf icesti2018 01031.html

9. Belajarforex. Create a trading system. [online] from http://belajarforex.com/huntingfox/4-create-a-trading-system.html (n.d.). [Accessed on August 20, 2020].

10. Facebook developers. Access tokens. [online] from https://developers.facebook.com/ docs/facebook-login/access-tokens/ (n.d). [Accessed on August 20, 2020].

11. Github. Facebook-php-sdk. [online] from https://github.com/facebook/facebook-phpsdk (n.d). [Accessed on August 20, 2020]. 